\title{
Maxillary Osteomyelitis Caused by Apophysomyces Variabilis - Emerging Trends
}

\author{
Neeraj Sharma ${ }^{1 *}$, Hemant Batra ${ }^{1}$, Manjula Mehta ${ }^{1}$, Jagdish Chander $^{2}$ \\ ${ }^{1}$ Dr. Hsj Institute of Dental Sciences, Panjab University, Chandigarh 160014, India - Department of Oral Medicine and \\ Radiology, sector 25 Panjab University Chandigarh, Chandigarh 160014, India; ${ }^{2}$ Govt Medical College and Hospital, Sector \\ 32 - Microbiology, Chandigarh, India
}

\begin{abstract}
Citation: Sharma N, Batra H, Mehta M, Chander J. Maxillary Osteomyelitis Caused by Apophysomyces Variabilis - Emerging Trends. OA Maced J Med Sci. 2014 Jun 15; 2(2):303308. http://dx.doi.org/10.3889/oamjms.2014.051

Key words: Osteomyelitis; mucormycete; Apophysomyces variabilis; fungal diseases; zygomycosis.

"Correspondence: Prof. Neeraj Sharma. Dr. Hsj Institute of Dental Sciences, Department of Oral Medicine and Radiology, sector 25, Panjab University, Chandigarh 160014, India. Phone: +917696008656.

E-Mail: neerajsh2003@gmail.com

Received: 26-Nov-2013; Revised: 06-Feb2014; Accepted: 08-Feb-2014; Online first: 24-Apr-2014

Copyright: () 2014 Sharma et al. This is an open-access article distributed under the terms of the Creative Commons Attribution License, which permits unrestricted use, distribution, and reproduction in any medium, provided the original author and source are credited.

Competing Interests: The authors have declared that no competing interests exist.
\end{abstract}

\begin{abstract}
In the maxillofacial skeleton, chronic osteomyelitis is more often observed in the mandible than maxilla. Maxillary osteomyelitis is rare because of its rich blood supply. It is usually seen in individuals with impaired immune response, uncontrolled diabetes and hospitalized patients. It can be caused by bacterial, fungal or viral infections. We report a rare case of maxillary osteomyelitis caused by an emerging mucormycete, Apophysomyces variabilis.
\end{abstract}

\section{Introduction}

Osteomyelitis of maxilla is a very rare pathology, the gravity of which was appreciated by Hippocrates as long ago as the 5th century BC [1]. It is seen less frequently because maxillary blood supply is more extensive as compared to the mandible. Thin cortical plates and a relative paucity of medullary tissue in the maxilla preclude confinement of infections within bone and permit the dissipation of edema and pus into the soft tissues and the paranasal sinuses [2].

The pathogenesis of osteomyelitis may be induced either by hematogenous origin or by dissemination of local infections, and its treatment involves removal of bone sequestra, lesion debridement, and bone decortication associated with systemic antibiotic therapy [3, 4]. The occurrence, type, severity and clinical prognosis of osteomyelitis depend upon several factors, including the characteristics and virulence of the infecting pathogen, host immune response and source of infection [5]. However, in some patients, these infections are refractory to surgery and antibiotic therapy [3], requiring an adequate microbiological diagnosis $[4,6]$.

In most of the cases the causative organism is Staphylococcus aureus or Staphylococcus epidermidis. Occasionally, anaerobic or microaerophilic cocci and Gram-negative organisms such as Klebsiella, Pseudomonas and Proteus spp., are found in association with the other pathogens. Fungal osteomyelitis is mostly caused by Aspergillus, Mucor spp [7]. Here, we report a case of bilateral maxillary osteomyelitis caused by Apophysomyces variabilis. 


\section{Case report}

A 32 year old male reported to the department of oral medicine and radiology with a six-month history of a non-healing wound in the maxilla. There was a history of pain in upper right back area seven months ago. He went to a private dentist who extracted his maxillary right third molar. There was no history of fever, purulent discharge, paraesthesia or foul odor.

The patient reported that even after extraction there was no relief from pain but instead it had started radiating to the anterior region of right maxilla. The site of extraction did not heal even after taking repeated courses of antibiotics. Patient then went to the private hospital where a CT scan was taken. He was then treated with empiric intravenous antibiotics for a period of 14 days and oral antibiotics thereafter for a period of 1 month. There was slight relief from pain.

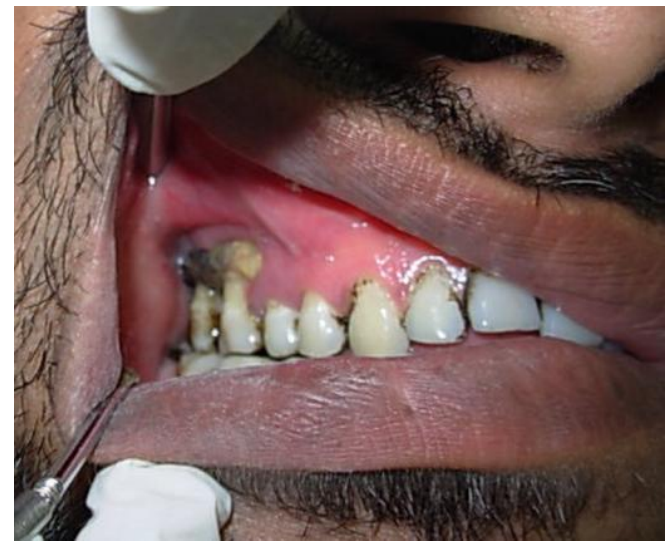

Figure 1: Intra oral view showing exposed necrotic bone in 16, 17 region.

At the time of presentation to our hospital, the medical history of the patient was non-contributory and the vital signs were stable. There were no risk factors for human immunodeficiency virus infection or tuberculosis. A nasal twang was noticed in the patients' voice. Inspection revealed a diffuse irregular swelling on the both sides of the maxilla.

Intra oral examination revealed a mucosal defect in the right maxillary molar region, with exposed necrotic bone in 16, 17 region (Figure 1). All maxillary teeth (17-27) were mobile. The right maxillary third molar was missing. Spacing was noticed between 11 and 21. Diffuse swelling was noticed on the hard palate (Figure 2).

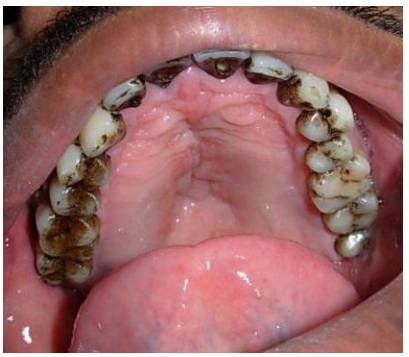

Figure 2: Edema seen over the palate.

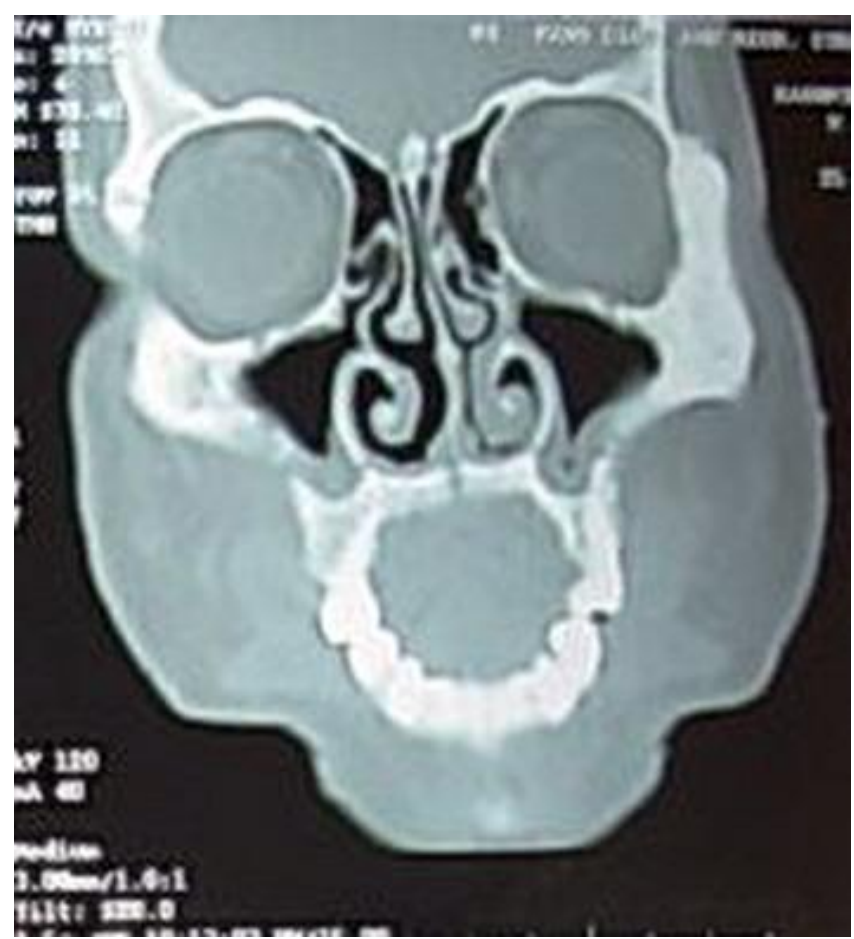

Figure 3: Past CT scan showing mid palatal dehiscence and break in the continuity of the lateral and medial walls of the bilateral maxillary sinus in the affected area.

Past medical records were assessed. The past CT scan (taken 5 months back) showed dehiscence of the mid-palatal suture (Figure 3) and break in the continuity of the lateral and medial walls of both maxillary sinuses. Polyps were also noticed in bilateral sinuses. The patient was asked to undergo a new set of investigations including CT scan, biochemical investigations, biopsy, and culture sensitivity.

The present CT scan showed marked destruction of maxillary alveoli with enhancing soft tissue density extending into bilateral maxillary sinuses and left nasal cavity. Bony resorption of the posterolateral wall of bilateral maxillary sinuses was also evident (Figure 4, 5).

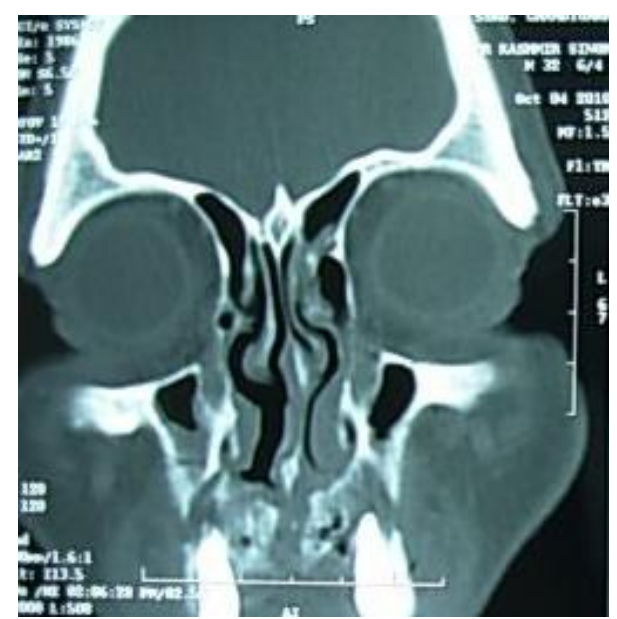

Figure 4: New CT scan showing marked destruction of the palate (coronal view) 
Biochemical investigations revealed normal blood sugar levels. Fasting blood sugar was $90 \mathrm{mg} / \mathrm{dl}$ \& postprandial blood sugar level was $138 \mathrm{mg} / \mathrm{dl}$. Routine blood investigations revealed - hemoglobin 14

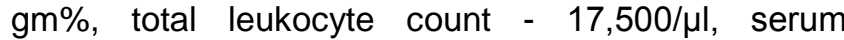
electrolytes:- total calcium - $10 \mathrm{mg} / \mathrm{dl}$, chloride - 98 $\mathrm{mEq} / \mathrm{l}$, potassium - $4.2 \mathrm{mEq} / \mathrm{l}$, sodium-138 mEq/l and creatinine $-1 \mathrm{mg} / \mathrm{dl}$.

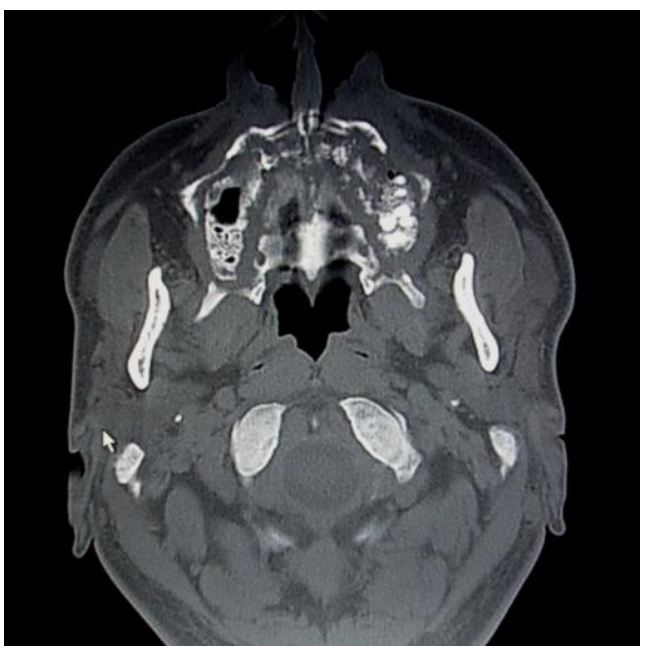

Figure 5: Axial view showing osteolytic lesion involving bilateral maxilla.

Among the clinical differential diagnosis we considered osteomyelitis (fungal) secondary to traumatic extraction, pre-existing fungal sinusitis which was now involving the surrounding bone, squamous cell carcinoma of maxillary sinus, malignant salivary gland tumor arising from the accessory glands of the palate, Wegener's granulomatosis, bone necrosis as an extension of infections such as acute necrotizing ulcerative gingivitis (ANUG) from the gingiva to bone.

A biopsy of the involved tissue was taken. Hard tissue specimen along with the adjacent soft tissue was excised under local anesthesia and sent for histopathological examination. The culture and sensitivity tests were also performed.

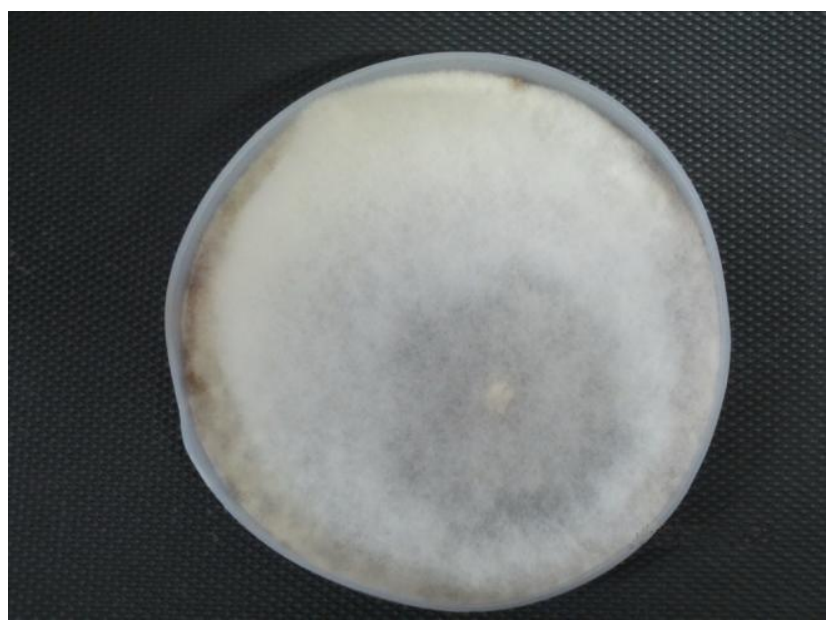

Figure 6: Floccose, white, fungal colonies of a mucomycete on Sabouraud dextrose agar.
Routine bacteriological cultures of the debrided tissue yielded Pseudomonas aeruginosa and Staphylococcus aureus. Cultures of debrided tissue on Sabouraud dextrose agar containing chloramphenicol and on Sabouraud dextrose agar containing chloramphenicol and cycloheximide yielded floccose, white to dirty-white, fast-growing fungal colonies of a mucormycete (Figure 6). The plates were incubated at $37^{\circ} \mathrm{C}$ in the dark for 7 days. Direct microscopy showed evidence of broad, aseptate, or sparsely septate ribbon-like hyphae with right-angled branching in stained sections of tissue specimens. The apophyses were dark, funnel or champagne glass shaped (Figure 7). The isolates were identified as Apophysomyces elegans Complex on morphological basis. The mold did not sporulate when subcultured on several routinely used mycological media.

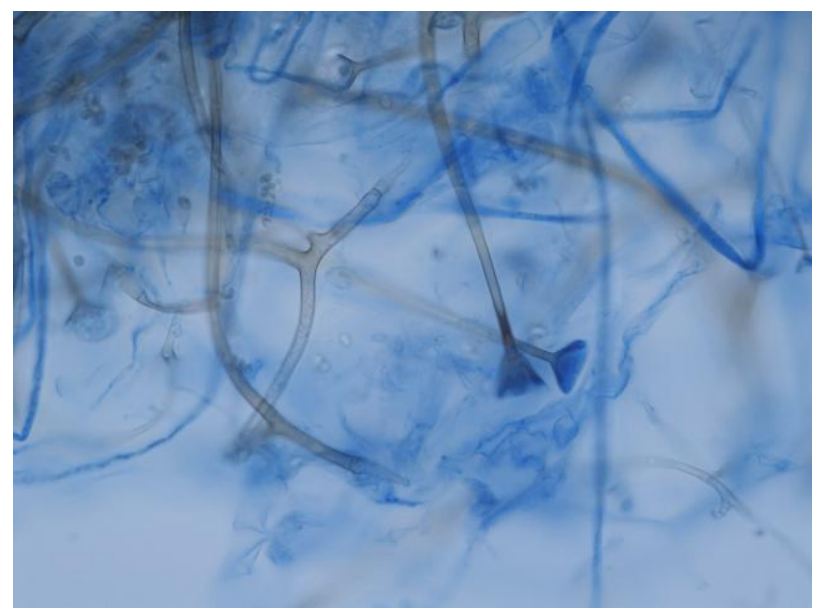

Figure 7: Lactophenol cotton blue staining of culture isolate demonstrating sporangiophores terminating in funnel-shaped apophyses. (magnification, $x$ 400).

The isolate was then sent to the Universitat Rovira i Virgili (Reus, Spain) for molecular analysis and was found to be Apophysomyces variabilis. (Gene bank accession no for the isolate is HE792980).

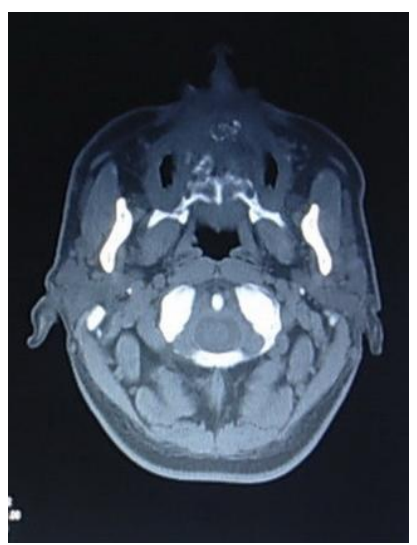

Figure 8: Post operative CT scan after debridement of the lesion (axial view).

The final diagnosis of maxillary osteomyelitis caused by Apophysomyces variabilis was made. The lesion was surgically debrided under general 
anesthesia. He was started on amphotericin B $0.8 \mathrm{mg} / \mathrm{kg} /$ day intravenously (i.v.) for two weeks. It was slowly infused over 4-6 hours and blood urea and creatinine levels were monitored as the drug can cause renal toxicity. Cefepime (i.v.) $50 \mathrm{mg} / \mathrm{kg}$ body weight 12 hourly was also prescribed for ten days. CT scan was repeated again after debridement (Figure 8).

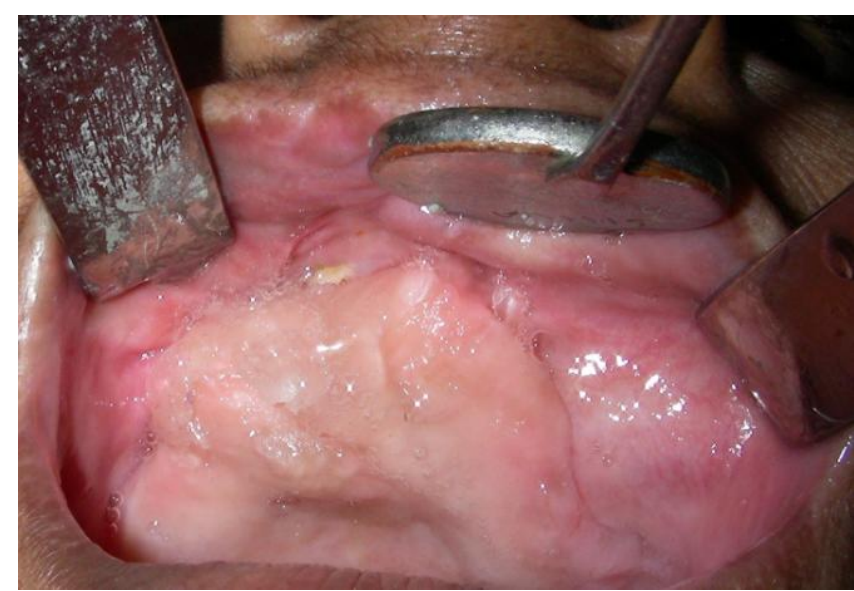

Figure 9: Post operative clinical photograph.

Follow-up clinical examination up to one year showed no evidence of persistent infection (Figure 9).

\section{Discussion}

Chronic osteomyelitis is a relapsing and persistent infection that evolves over months to years and is characterized by low-grade inflammation, presence of bone sequestra, new bone apposition and, sometimes fistulous tracts [8]. Odontogenic infection is the most common cause of osteomyelitis of the jaws, although other causes including injury, malnutrition, diabetes mellitus, malignant tumors, corticosteroid therapy, immunosuppressive drugs, leucopenia, chronic systemic and infectious diseases occurring in hypovascularized bone may be associated with this condition [9-11].

The most common presenting signs and symptoms of maxillary osteomyelitis include fever, headache, sinusitis, facial pain and swelling, mucosal ulceration and/or necrosis. Radiographic feature is an ill-defined osteolytic lesion with loss of lamina dura. Diagnosis is based on clinical picture revealing the invasive course of the disease and by culture and histopathology. In our case also, the clinical signs and radiographic features did not vary from the other usual causes of maxillary osteomyelitis. Routine culture on Sabouraud dextrose agar containing chloramphenicol helped to identify Apophysomyces species.

Apophysomyces is a member of the fungal class Mucormycetes, order Mucorales. Although mucormycetes are ubiquitous environmental pathogens, disease from Apophysomyces is rare. In a study it has been proved that $A$. elegans could not be isolated from hospital environment [12]. It is an emerging pathogen that, unlike the other members of Mucorales, has been reported to cause invasive cutaneous, renal, abdominal and rhino-orbitocerebral infections in immunocompetent individuals [13]. The infection typically follows traumatic implantation of the agent but may also result from inhalation of spores into the sinus $[14,15]$.

Excellent mycelial growth is seen on standard culture media (Sabouraud's dextrose medium). It grows as a floccose aerial mycelium, demonstrating white to grey confluent growth in 2 days on standard culture medium [16]. A special nutrient-deficient growth medium, a high temperature of incubation and prolonged incubation can be used to induce $A$. elegans isolates to sporulate [13, 16-18]. A. elegans is a thermotolerant fungus that is capable of rapid growth at $24-43^{\circ} \mathrm{C}$ in vitro $[10,19,20]$ and it is believed that this thermotolerance permits fungal proliferation in deep tissues. Although no specific virulence factors have yet been identified for this organism, it produces disease in much the same way as the other mucorales, with tissue and angio-invasion [16].

The $\mathrm{KOH}$ wet mount can also be done which demonstrates broad aseptate hyphae with rightangled branching. The growth obtained on culture are identified by colony characteristics, lactophenol cotton blue preparation and slide culture. For sporulation, agar flotation method is used i.e. one cm SDA agar blocks permeated with hyphae and accompanying aerial hyphae are cut and placed on surface of sterile water-yeast extract solution [21].

Apophysomyces elegans Complex now includes four species - Apophysomyces elegans, $A$. ossiformis, $A$. trapeziformis and $A$. variabilis - with genetic, physiological and morphological differences [21]. The differences in morphology of sporangiophores and sporangiospores correlate with the different phylogenetic species. The strains of $A$. variabilis show morphological diversity. The sporangiospores range from broadly clavate to ellipsoidal with flattening on one side. The strains of $A$. elegans, show ovoid, subspherical, broadly ellipsoidal to barrel shaped sporangiospores and are the largest among the different species in the complex. The sporangiospores of $A$. trapeziformis are trapezoidal and smaller while those of Apophysomyces ossiformis are thick-walled and clearly biconcave (bone-shaped) $[22,23]$.

Aggressive management including surgical intervention with or without medical therapy is required for the treatment of Apophysomyces spp. infections [14]. Surgical removal of infected tissue provides a more definitive treatment, while the response to amphotericin $B$ is variable at best. Liposomal encapsulation of amphotericin may facilitate deposition of the drug into the 
reticuloendothelial system and higher dose concentrations in the central nervous system, resulting in increased efficacy. It also appears to enhance delivery to fungi, infected organs, and phagocytes [24]. The toxicity is decreased significantly enabling higher dosages to be administered. Posaconazole, a new triazole, can be a good alternative in patients with amphotericin B associated toxicity. The initial dose of $200 \mathrm{mg}$ four times a day can be changed to $400 \mathrm{mg}$ twice a day once the patient is clinically stable and is tolerating the medication. Advantages of this medication are its oral formulation, a favorable safety profile, an adequate oral bioavailability and good tolerance, which makes it much easier to manage the patients on an ambulatory basis once they are clinically stable [25].

Although most cases of infection with $A$. variabilis fungi have been reported in India [14], infections with this fungus may have a wider distribution. A recent study demonstrated that this species represented $0.5 \%$ of fungi of the order Mucorales isolated from clinical samples in the United States [26]. Apophysomyces can cause cutaneous, renal, abdominal, rhino-orbito-cerebral and disseminated infections in the host. Furthermore, a high mortality rate and the fact that most of these infections involve otherwise healthy patients make this a serious infection.

In our case, along with Apophysomyces sp. we were also able to culture Staphylococcus aureus and Pseudomonas aeruginosa. Mixed anaerobic infections are common in osteomyelitis, especially in jaw infections. Several fastidious strictly anaerobic bacteria commonly present in the dental biofilm can be detected, suggesting that the source of infecting pathogens in osteomyelitis of the jaws is likely to be gingivitis, chronic periodontitis, previous dental extractions or endodontic treatments [4, 5, 27]. Also pathogens different from those of the oral microbiota may reach the bone tissues through transient bacteremia, which are common after surgical procedures or traumas [28, 29]. We could however not ascertain the route of fungal infection which could have been either iatrogenic (following extraction) or due to inhalation of spores.

Our patient's case stresses the relevance of Apophysomyces elegans Complex as an emerging mucormycosis especially in the tropical and subtropical regions. A lack of awareness about fungal infections in most centers of developing countries underestimates its importance. It is a requirement that in an immunocompetent patient with maxillary osteomyelitis from tropical areas, one should rule out Apophysomyces infection. While the clinical presentations, outcomes and treatment strategy of osteomyelitis caused by this fungus are similar to other infections, this emerging trend of fungal infection emphasizes the need to be cognizant that mucormycosis may occur in an immunocompetent host, especially following trauma or extraction.

\section{Acknowledgments}

The authors would like to thank Dr. Josep Guarro, Mycology Unit,MedicalSchooland IISPV, Universitat Rovira i Virgili, Reus, Spain for helping with the molecular analysis of the specimen.

\section{References}

1. Chadwick J and Mann W N translators (1950): Hippocrates' Medical Works, p. 238. Oxford: Blackwell.

2. Topazian RG, Goldberg MH, Hupp JR. Oral and maxillofacial infections $4^{\text {th }}$ edition. Philadelphia :WB Saunders Company 2002; p 215

3. Brook I, Frazier EH. Anaerobic osteomyelitis and arthritis in a military hospital: a 10-year experience. Am. J. Med. 1993; 94: 2-8.

4. Coviello V, Stevens MR. Contemporary concepts in the treatment of chronic osteomyelitis. Oral Maxillofac Surg Clin N Am 2007; 19: 523-534.

5. Brady BA, Leid JG, Costerton JW, Shirtliff ME. Osteomyelitis: clinical overview and mechanisms of infection persistence. Clin Microbiol Newsletter 2006; 28: 65-72.

6. Kim SG, Jang HS. Treatment of chronic osteomyelitis in Korea. Oral Surg Oral Med Oral Pathol Oral Radiol Endod 2001; 92: 394-398.

7. Burduk BK, Skorek A, Stankiewicz C, Dalke K.Chronic, recurrent, progressive osteomyelitis of the maxilla caused by Methicillin-resistant Staphylococcus epidermidis: a therapeutic dilemma. J Oral Maxillofac Surg 2010; 68:2012-2015.

8. Zuluaga, A.F.; Galvis, W.; Saldarriaga, J.G.; Agudelo, M.; Salazar, B.E.;Vesga, O. (2006). Etiologic diagnosis of chronic osteomyelitis. Arch Intern Med. 166, 95-100.

9. Prasad KC, Prasad SC, Mouli N, Agarwal S. Osteomyelitis in the head and neck. Acta Oto-Laryngol 2007; 127: 194-205.

10. Holland J. Emerging zygomycoses of humans: Saksenaea vasiformis and Apophysomyces elegans. Curr Top Med Mycol 1997; 8:27-34.

11. Brown AE. Overview of fungal infections in cancer patients. Semin Oncol 1990; 17:2-5.

12. Chakrabarti A, Das A, Sharma A, Panda N, Das S, Gupta KL and Sakhuja V. Ten years' experience in zygomycosis at a tertiary care centre in India. J Infect 2001; 42:261-266.

13. Wieden MA, Steinbronn KK, Padhye A, Ajello $L$ and Chandler FW. Zygomycosis caused by Apophysomyces elegans. J Clin Microbiol 1985; 22:522-526

14. Chakrabarti A, Ghosh A, Prasad GS, David JK, Gupta S, Das A, Sahkuja V, Panda NK, Singh SK, Das S and Chakrabarti T. Apophysomyces elegans: an emerging zygomycete in India. J Clin Microbiol 2003; 41:783-788.

15. Liang KP,Tleyjeh IM,Wilson WR, Roberts GD,Temesgen Z. Rhino-orbitocerebral mucormycosis caused by Apophysomyces elegans. JClinMicrobiol. 2006;44:892-898.

16. Meis JF and Chakrabarti A. Changing epidemiology of an emerging infection: zygomycosis. Clin Microbiol Infect 2009; 15 (Suppl. 5): 10-14

17. Huffnagle KE, Southern PM, Byrd CT, Gander RM Apophysomyces elegans as an agent of zygomycosis in a patient following trauma. J Med Vet Mycol 1992; 30: 83-86.

18. Padhye AA, Ajello L. Simple method of inducing sporulation by Apophysomyces elegans and Saksenaea vasiformis. J Clin Microbiol 1988; 26:1862-1863.

19. Meis JF, Kullberg BJ, Pruszczynski M, Veth RPH. Severe 
osteomyelitis due to the zygomycete Apophysomyces elegans. J Clin Microbiol 1994; 32: 3078-3081.

20. Misra PC, Srivastava KJ, Lata K. Apophysomyces, a new genus of the Mucorales. Mycotaxon 1979; 8: 377-382.

21. Padhye AA, Ajello L.12. Simple method of inducing sporulation by Apophysomyces elegans and Saksenaea vasiformis. J Clin Microbiol 1988;26: 1862-3.

22. Marisa Z. R. Gomes, Russell E. Lewis and Dimitrios P. Kontoyiannis. Mucormycosis caused by unusual Mucormycetes, Non-Rhizopus, -Mucor, and -Lichtheimia Species Clin. Microbiol. Rev. 2011, 24:411-445.

23. Alvarez E, Stchigel AM, Cano J, Sutton DA, Fothergill AW, Chander J, Salas V, Rinaldi MG and Guarro J. Molecular phylogenetic diversity of the emerging mucoralean fungus Apophysomyces: proposal of three new species. Rev. Iberoam. Micol. 2010;27:80-89.

24. Yohai RA, Bullock JD, Aziz AA, Marker RJ. Survival factorsin rhino-orbital-cerebral mucormycosis. Surv Ophthalmol 1994; 39:3-22.

25. Kok J, Gilroy N, Halliday C, Lee OC, Novakovic D, Kevin P Chen S. Early use of posaconazole in the successful treatment of rhino-orbital mucormycosis caused by Rhizopus oryzae. $\mathrm{J}$ Infect 2007;55:33-36

26. Alvarez E, Sutton DA, Cano J, Fothergill AW, Stchigel A, Rinaldi MG, et al. Spectrum of zygomycete species identified in clinically significant specimens in the United States. J Clin Microbiol. 2009;47:1650-6.

27. O'Sullivan D, King $P$ and Jagger $D$. Osteomyelitis and pathological mandibular fracture related to a late implant failure: a clinical report. J Prosthet Dent 2006; 9:106-10.

28. Takai S, Kuriyama T, Yanagisawa M, Nakagawa K, Karasawa $\mathrm{T}$. Incidence and bacteriology of bacteremia associated with various oral and maxillofacial surgical procedures. Oral Surg Oral Med Oral Pathol Oral Radiol Endod 2005; 99: 292-298.

29. Calhoun KH, Shapiro RD, Stiernberg CM, Calhoun JH, Mader JT. Osteomyelitis of the mandible. Arch Otolaryngol Head Neck Surg.1988; 114: 1157-1162. 